\title{
A necessary and sufficient BEM/BIEM for two-dimensional elasticity problems
}

\author{
J. T. Chen ${ }^{1,2}$, W. S. Huang ${ }^{1}$, Y. T. Lee ${ }^{1} \&$ S. K. Kao ${ }^{1}$ \\ ${ }^{1}$ Department of Harbor and River Engineering, \\ National Taiwan Ocean University, Taiwan \\ ${ }^{2}$ Department of Mechanical and Mechatronic Engineering, \\ National Taiwan Ocean University, Taiwan
}

\begin{abstract}
In the development of finite element method (FEM), the patch test is required. We may wonder whether we need any special test for the boundary element method (BEM). A sufficient and necessary boundary integral equation method (BIEM) to ensure a unique solution is our concern. In this paper, we revisit this issue for the interior two-dimensional (2-D) elasticity problem and investigate the equivalence of solution space between the integral equation and the partial differential equation. Based on the degenerate kernel and the eigenfunction expansion, the range deficiency of the integral operator of the single-layer potential for the solution space in the degenerate-scale problem for the 2-D elasticity in the BIEM is analytically studied. Following the Fichera's idea, we enrich the conventional BIEM by adding constants and corresponding constraints. In addition, we introduce the concept of modal participation factor (MPF) to examine whether the adding term of the rotation is required for interior simply-connected problems. Finally, a simple example of the degenerate-scale problem containing an elliptical boundary subjected to various boundary conditions of the rigid body translation and rotation for 2-D elasticity problems was demonstrated by using the necessary and sufficient BIEM.
\end{abstract}

Keywords: boundary integral equation, boundary element method, Fichera's method, degenerate scale, degenerate kernel. 


\section{Introduction}

It is well known that the boundary integral equation method (BIEM) and the boundary element method (BEM) are widely used to solve engineering problems. Nevertheless, mathematical models of the integral equation for engineering problems are not equivalent for the solution space to those of the partial differential equations (PDE) as pointed out by Feng [1] and $\mathrm{Yu}$ [2]. A degenerate scale [3] as a well-known $\Gamma$ contour problem in the BEM has been noticed for a long time. In the past experience of solving the Laplace problem [4-6], the main key of the BEM was the fundamental solution containing the base of $\ln r$. Even though in the 2-D elasticity problem, the degenerate scale also exists in the BEM/BIEM [7-11]. The occurring mechanisms of two problems, Laplace and Navier, are similar due to the $\ln r$ term. However, there are two degenerate scales for elasticity problems instead of only one for Laplace problems. In the literature, there are various regularized methods to deal with the degenerate-scale problem for 2-D Laplace problems [3, 4, 12]. Recently, Chen et al. [3, 13] used the Fichera's method by adding the free constant term and the corresponding constraint to transform an illposed system to a well-posed system through the analytical derivation by using the degenerate kernel and Fourier series. A necessary and sufficient BIE obtained from this approach was derived to avoid the non-uniqueness solution for Laplace problems. However, it is interesting that $\mathrm{Hu}$ et al. [14] and $\mathrm{He}$ et al. [10] who proposed that both a translation term and a rotation term were required for 2-D elasticity problems. It is different from the Fichera's method [15] which adds the free constant and the corresponding constraint. Therefore, we may wonder whether the rotation term is needed or not for the interior problem.

In this paper, we use the degenerate kernel instead of the closed-form fundamental solution to derive the analytical degenerate scale and field response for the isotropic elasticity problem containing an elliptical boundary. By way of the analytical derivation, the range deficiency of the solution space is found and we find that a constant term could not appear in case of a degenerate scale. It means that the integral equation is not equivalent to the partial differential equation for the solution space. In the linear algebraic system, the singular value decomposition (SVD) and the modal participation factor (MPF) are used to study the contribution of the singular vector corresponding to the zero singular value for various boundary conditions. Through the analytical derivation and numerical implementation, we find that the extra rotation term in $[10,16]$ may not be needed for degenerate-scale problems. It is well known that FEM code was examined by using the patch test to solve a simple example of constant strain case. Similarly, the BEM code should be checked by using a more simple solution of the rigid body translation and rotation. Since the conventional BEM/BIEM fails to solve the degenerate-scale problem, we propose the necessary and sufficient $\mathrm{BEM} / \mathrm{BIEM}$ to deal with this problem. An example of the degenerate-scale problem containing an elliptical boundary for 2-D elasticity is demonstrated by using the necessary and sufficient BEM/BIEM. 


\section{Problem statement and formulation}

For simplicity, the medium is considered to be linearly elastic, isotropic and homogenous. The governing equation is the Navier equation as follows:

$$
(\lambda+G) \nabla(\nabla \cdot \underset{\sim}{u}(\mathbf{x}))+G \nabla^{2} \underset{\sim}{u}(\mathbf{x})=\underset{\sim}{0}, \mathbf{x} \in D,
$$

where $\underset{\sim}{u}(\mathbf{x})$ is the displacement of the field point $\mathbf{x}, D$ is the domain of interest, $\nabla^{2}$ is the Laplace operator, $\lambda$ and $G$ are the Lamé constants for the isotropic elasticity. The integral representation of single-layer potential for the solution yields

$$
u_{i}(\mathbf{x})=\int_{B} U_{i j}(\mathbf{x}, \mathbf{s}) \alpha_{j}(\mathbf{s}) d B(\mathbf{s}), \mathbf{x} \in D,
$$

where $U_{i j}(\mathbf{x}, \mathbf{s})$ is the fundamental solution for the displacement response of the $i$ th direction at the field point $\mathbf{x}$ due to a concentrated load of the $j$ th direction at the source $\mathbf{s}$ and $\alpha_{j}(\mathbf{s})$ is the unknown boundary density. The explicit form of $U_{i j}(\mathbf{x}, \mathbf{s})$, or the so-called Kelvin solution, is

$$
U_{i j}(\mathbf{x}, \mathbf{s})=-\frac{1}{8 \pi G(1-v)}\left(\kappa \delta_{i j} \ln r-\frac{y_{i} y_{j}}{r^{2}}\right),
$$

where $\delta_{i j}$ is the Kronecker delta, $v$ is the Poisson ratio $(v=\lambda / 2(\lambda+G))$, $\kappa=3-4 v, r \equiv|\mathbf{x}-\mathbf{s}|, y_{i}=x_{i}-s_{i}, i=1,2$ and $j=1,2$ for the plane elasticity.

To provide a test for the BEM, we design an exact solution containing the constant term and the rotation term as shown below:

$$
\begin{aligned}
& u_{1}(\mathbf{x})=1+x_{1}\left(\cos \left(\theta_{r}\right)-1\right)-x_{2} \sin \left(\theta_{r}\right), \mathbf{x} \in D, \\
& u_{2}(\mathbf{x})=1+x_{1} \sin \left(\theta_{r}\right)+x_{2}\left(\cos \left(\theta_{r}\right)-1\right), \mathbf{x} \in D,
\end{aligned}
$$

where $\mathbf{x}\left(x_{1}, x_{2}\right)$ is the field point and $\theta_{r}$ is the angle of the rigid body rotation. The boundary conditions are

$$
f_{i}(\mathbf{x})=u_{i}(\mathbf{x}), \mathbf{x} \in B, i=1,2,
$$

where $B$ is the boundary of domain.

\section{Derivation of the analytical solution for an elliptical domain by using the BIEM in conjunction with the degenerate kernel}

For an elliptical-domain problem, we derive the analytical solution for the case of the rigid body translation and the rigid body rotation by using the BIEM in conjunction with the degenerate kernel [17] in terms of the elliptic coordinates. The distribution of field points is shown in figure 1 . The source point $\mathbf{s}$ and the collocation point $\mathbf{x}$ are expressed by $(\bar{\xi}, \bar{\eta})$ and $(\xi, \eta)$, respectively. The 
unknown boundary densities $\alpha_{j}(\mathbf{s})$ and the given boundary conditions $f_{i}(\mathbf{x})$ along the boundary can be expressed by using the eigenfunction expansion as shown below:

$$
\begin{gathered}
\alpha_{j}(\mathbf{s})=\frac{1}{J_{s}}\left(a_{0}^{(j)}+\sum_{n=1}^{\infty} a_{n}^{(j)} \cos (n \bar{\eta})+b_{n}^{(j)} \sin (n \bar{\eta})\right), \quad 0 \leq \bar{\eta} \leq 2 \pi, \quad j=1,2, \\
f_{i}(\mathbf{x})=p_{0}^{(i)}+\sum_{n=1}^{\infty} p_{n}^{(i)} \cos (n \eta)+q_{n}^{(i)} \sin (n \eta), \quad 0 \leq \eta \leq 2 \pi, \quad i=1,2,
\end{gathered}
$$

where, $a_{0}^{(j)}, a_{n}^{(j)}$ and $b_{n}^{(j)}$, are unknown coefficients, the coefficients, $p_{0}^{(i)}, p_{n}^{(i)}$ and $q_{n}^{(i)}$, are determined by the specified boundary conditions, and the Jacobian term is $J_{\mathrm{s}}=c \sqrt{\sinh ^{2} \bar{\xi}+\sin ^{2} \bar{\eta}}$. By using the degenerate kernel in terms of the elliptic coordinates [17], the coefficients of the eigenfunction representation of the boundary densities could be obtained as follows [18]:

$$
\begin{aligned}
& a_{0}^{(1)}=\frac{8 G(1-v)}{-2 \kappa\left(\ln \frac{a+b}{2}\right)+\frac{2 a}{a+b}} p_{0}^{(1)}, \\
& a_{n}^{(1)}=8 G(1-v) \frac{\left(\frac{\sinh (n \bar{\xi})}{\sinh (2 \bar{\xi}) e^{-n \bar{\xi}}}-\frac{n}{2 \kappa}\right) p_{n}^{(1)}+\frac{n}{2 \kappa} q_{n}^{(2)}}{\left(\frac{\kappa}{n} \frac{\sinh (2 n \bar{\xi})}{\sinh (2 \bar{\xi})}-1\right)}, n \geq 1 \text {, } \\
& b_{n}^{(1)}=8 G(1-v) \frac{\left(\frac{\cosh (n \bar{\xi})}{\left.\sinh (2 \bar{\xi}) e^{-n \bar{\xi}}+\frac{n}{2 \kappa}\right) q_{n}^{(1)}+\frac{n}{2 \kappa} p_{n}^{(2)}}\right.}{\left(\frac{\kappa}{n} \frac{\sinh (2 n \bar{\xi})}{\sinh (2 \bar{\xi})}+1\right)}, n \geq 1 \text {, } \\
& a_{0}^{(2)}=\frac{8 G(1-v)}{-2 \kappa\left(\ln \frac{a+b}{2}\right)+\frac{2 b}{a+b}} p_{0}^{(2)}, \\
& a_{n}^{(2)}=8 G(1-v) \frac{\left(\frac{\sinh (n \bar{\xi})}{\sinh (2 \bar{\xi}) e^{-n \bar{\xi}}}+\frac{n}{2 \kappa}\right) p_{n}^{(2)}+\frac{n}{2 \kappa} q_{n}^{(1)}}{\left(\frac{\kappa}{n} \frac{\sinh (2 n \bar{\xi})}{\sinh (2 \bar{\xi})}+1\right)}, n \geq 1 \text {, } \\
& b_{n}^{(2)}=8 G(1-v) \frac{\left(\frac{\cosh (n \bar{\xi})}{\sinh (2 \bar{\xi}) e^{-n \bar{\xi}}}-\frac{n}{2 \kappa}\right) q_{n}^{(2)}+\frac{n}{2 \kappa} p_{n}^{(1)}}{\left(\frac{\kappa}{n} \frac{\sinh (2 n \bar{\xi})}{\sinh (2 \bar{\xi})}-1\right)}, n \geq 1 \text {. }
\end{aligned}
$$


where $a$ and $b$ are the lengths of the semi-major axis and the semi-minor axis of the elliptical domain, respectively. It is found that the coefficients of the constant term ( $a_{0}^{(1)}$ of equation (9) and $a_{0}^{(2)}$ of equation (12)) could not be determined due to the zero denominator when a degenerate scale occurs,

$$
2 \kappa\left(\ln \frac{a+b}{2}\right)=\frac{2 a}{a+b},
$$

or

$$
2 \kappa\left(\ln \frac{a+b}{2}\right)=\frac{2 b}{a+b} .
$$

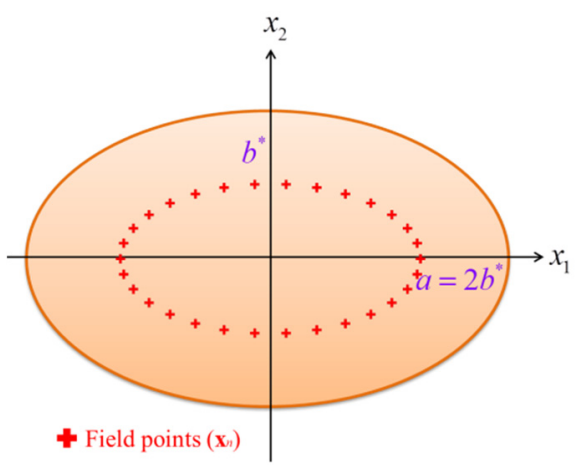

Figure 1: The sketch of the problem.

However, the coefficients of the rotation term ( $a_{1}^{(1)}$ of equation $(10), b_{1}^{(1)}$ of equation (11), $a_{1}^{(2)}$ of equation (13) and $b_{1}^{(2)}$ of equation (14)) could be determined for any scale. That is to say that the single-layer integral operator is range deficient by the constant term only. After derivation of the above elliptical case, we found that the range of the integral operator is only short of the constant term instead of the rotation term when the size of the domain is a degenerate scale. According to the Fredholm alternative theorem, we obtain the infinite solution when $\int_{B} f_{i}(\mathbf{x}) d B(\mathbf{s})=0$, or no solution when $\int_{B} f_{i}(\mathbf{x}) d B(\mathbf{s}) \neq 0$. In other words, the conventional single-layer potential BIEM is not sufficient (infinite solutions) and not necessary (no solution) to ensure a unique solution for a degenerate-scale problem.

\section{Regularized methods for a degenerate-scale problem}

\subsection{Necessary and sufficient BEM/BIEMs for 2-D elasticity problems}

According to the above analytical result, it is found that only the coefficients of the constant term could not be determined due to the zero denominator when the 
size of the domain is a degenerate scale of equation (15) and equation (16). Range deficiency by a constant term for the solution space appears. However, the coefficients of the rotation term and other higher order terms can be determined for any size of the elliptical domain. Therefore, the range deficiency of the BIE only occurs in the constant term in case of a degenerate scale. Following the past experience of solving the Laplace problem, we introduce Fichera's idea to solve the degenerate-scale problem. We enrich the range of the integral operator by adding constants $c_{i}$ and the corresponding constraints to enforce the indeterminate constant term of the boundary densities to be zero. The necessary and sufficient BEM/BIEM could be written as follows:

$$
\begin{gathered}
u_{i}(\mathbf{x})=\int_{B} U_{i j}(\mathbf{x}, \mathbf{s}) \alpha_{j}(\mathbf{s}) d B(\mathbf{s})+c_{i}, \mathbf{x} \in D, \\
\int_{B} \alpha_{i}(\mathbf{s}) d B(\mathbf{s})=0 .
\end{gathered}
$$

Equation (17) and equation (18) are different from Hu's necessary and sufficient BIEs [14] which contains a more rotation term and one more constraint as shown below:

$$
\begin{gathered}
u_{i}(\mathbf{x})=\int_{B} U_{i j}(\mathbf{x}, \mathbf{s}) \alpha_{j}(\mathbf{s}) d B(\mathbf{s})+c_{i}+\omega \varepsilon_{i j k} \underset{\sim}{x_{j}} e_{k}, \mathbf{x} \in D, \\
\int_{B} \alpha_{j}(\mathbf{s}) d B(\mathbf{s})=0, \mathbf{s} \in B, j=1,2, \\
\int_{B} \alpha(\mathbf{s}) \times \mathbf{s} d B(\mathbf{s})=0, \mathbf{s} \in B,
\end{gathered}
$$

where $\omega$ is the unknown rotation term and $\underset{\sim}{e}=(0,0,1)$ for the 2-D case.

\subsection{Modal participation factor to examine the numerical instability}

According to the analytical derivation, we can find that the adding rotation term in Hu's BIEs may not be necessary for a degenerate-scale problem of 2-D elasticity. In the linear algebraic system, we employed the SVD technique to find the MPF of the near-zero minimum singular vector due to a degenerate scale. By using the SVD technique, the influence matrix $\mathbf{A}$ could be decomposed as

$$
\mathbf{A}=\Phi \Sigma \Psi^{\mathrm{T}}=\sum_{n} \sigma_{n}{\underset{\sim}{n}}_{\sim} \underset{\sim}{\psi_{n}^{\mathrm{T}}},
$$

where ${\underset{\sim}{n}}_{n}, \psi_{\sim}$ and $\sigma_{n}$ are the $n$th left singular vector, the $n$th right singular vector and the $n$th singular value, respectively. When the rank deficiency exists in the influence matrix, the minimum singular value approaches zero. After the boundary element discretization, equation (2) could be expressed as follows:

$$
\mathbf{U} \underset{\sim}{\alpha}=\underset{\sim}{f},
$$

where $\underset{\sim}{\alpha}$ and $\underset{\sim}{f}$ are the vectors of the boundary densities and the boundary conditions, respectively. The boundary densities and boundary conditions could be expanded by 


$$
\begin{aligned}
& \underset{\sim}{\alpha}=\sum_{n} \gamma_{n} \underset{\sim}{\psi_{n}}, \\
& \underset{\sim}{f}=\sum_{n} \beta_{n} \phi_{\sim} .
\end{aligned}
$$

Following the MPF in the structural dynamics, the MPF $\left(\beta_{n}\right)$ with respect to $\phi_{n}$ is defined by

$$
\beta_{n}=\phi_{\sim}^{\mathrm{T}} \underset{\sim}{f} .
$$

According to equations (43), (45) and (46), equation (44) could be expressed as follows:

$$
\sigma_{n} \gamma_{n}=\beta_{n} .
$$

The coefficients of the boundary densities could be expressed as shown below:

$$
\gamma_{n}=\beta_{n} / \sigma_{n} .
$$

When the influence matrix $\mathbf{U}$ is a singular matrix, the minimum singular value is near zero in the numerical implementation. Therefore, the coefficients of the boundary densities could not be easily determined due to the numerical instability. Once $\beta_{n}$ is zero, it means that there is no contribution for the numerical instability of $\gamma_{n}$ for the mode of near-zero singular value $\left(\sigma_{n}\right)$.

\section{An illustrative example}

For a degenerate-scale problem containing an elliptical boundary, two different degenerate scales are given in equation (15) and equation (16). The exact solution of displacement field is given by equation (4) and equation (5), subjected to the boundary conditions of equation (6), where the angle of rotation $\left(\theta_{r}\right)$, the Lamé constants $G$ and $v$ are given as $30^{\circ}, 1.0$ and 0.25 , respectively.

When the size of the elliptical domain is a degenerate scale, the constant terms could not be determined according to the analytical derivation as given in equation (9) and equation (12). Therefore, the constant term of the solution space is deficient. In figure 2 and figure 3, the numerical result by using the conventional BEM is also unreasonable, where the number of boundary elements is 100 , the minimum singular values of the influence matrix are $2.9 \times 10^{-6}$ and $3.6 \times 10^{-6}$ corresponding to two degenerate scales, $b=0.930785701$ and $b=0.787891333$, respectively. Since the reasonable displacement in the degenerate-scale problems could not be obtained by using the conventional BEM/BIEM, we employed the Fichera's idea and proposed an enriched formulation by adding constants and corresponding constraints to improve the conventional BEM/BIEM.

This regularization approach, the necessary and sufficient BEM/BIEM, was also used to solve the problem. The results are more accurate than those obtained by using the conventional BEM/BIEM as shown in figure 2 and figure 3 . We can 


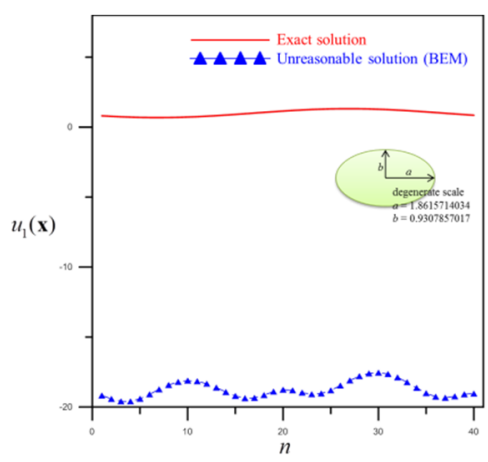

(a) The displacement $u_{1}(\mathbf{x})$ by using the conventional BEM

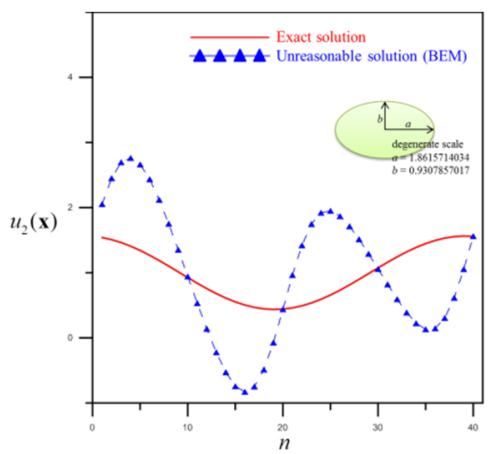

(b) The displacement $u_{2}(\mathbf{x})$ by using the conventional BEM

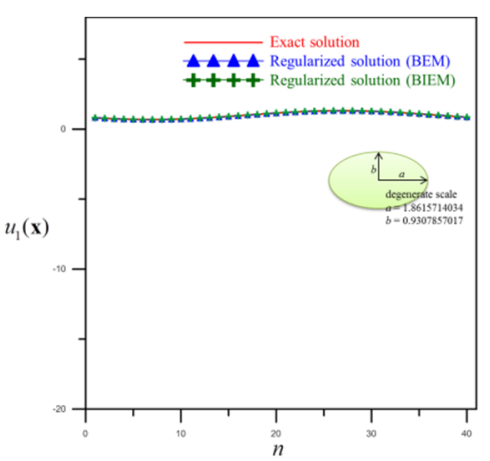

(c) The displacement $u_{1}(\mathbf{x})$ by using the present method

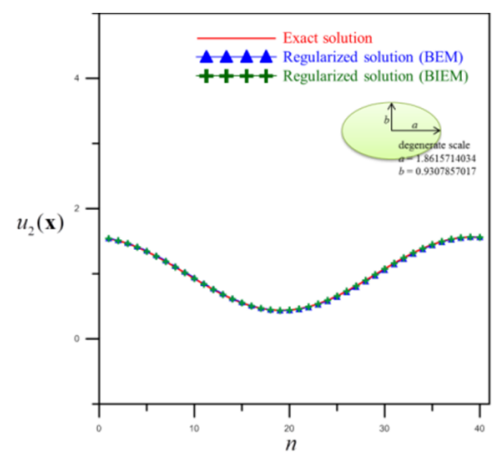

(d) The displacement $u_{2}(\mathbf{x})$ by using the present method

Figure 2: The results of the degenerate-scale case $(b=0.9307857017)$ for an elliptical domain.

successfully solve the degenerate-scale problem even if the rotation term is not enriched in the regularized method.

In order to understand the MPF for various boundary conditions, we examine two cases of an ellipse. One is the rigid body translation as given by

$$
f_{i}(\mathbf{x})=1, i=1,2
$$

and the other is the rigid body rotation as given by

$$
\begin{aligned}
& f_{1}(\mathbf{x})=x_{1}\left(\cos \left(\theta_{r}\right)-1\right)-x_{2} \sin \left(\theta_{r}\right) \\
& f_{2}(\mathbf{x})=x_{1} \sin \left(\theta_{r}\right)+x_{2}\left(\cos \left(\theta_{r}\right)-1\right)
\end{aligned}
$$

where the angle of rotation is $30^{\circ}$, the size of domain is a degenerate scale $(b=$ $0.787891333)$. The results for various boundary conditions by using the BEM are 


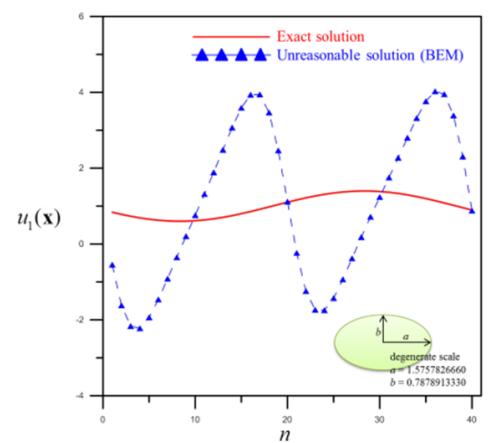

(a) The displacement $u_{1}(\mathbf{x})$ by using the conventional BEM

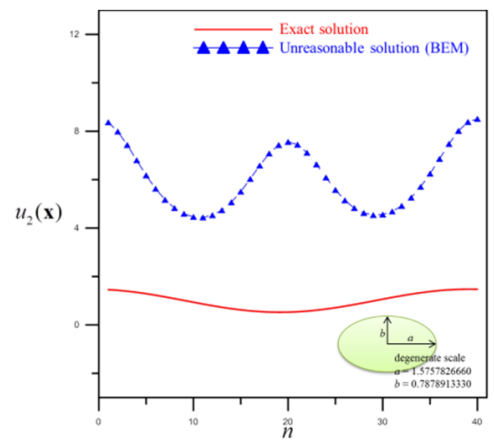

(b) The displacement $u_{2}(\mathbf{x})$ by using the conventional BEM

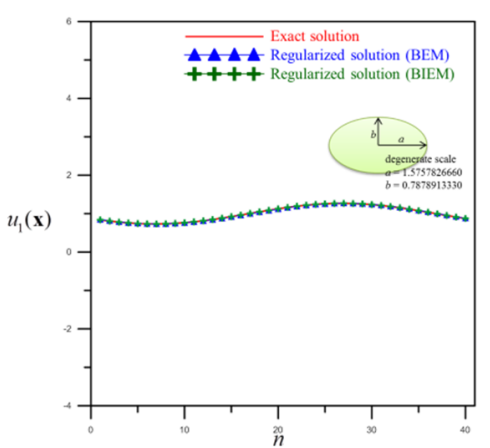

(c) The displacement $u_{1}(\mathbf{x})$ by using the present method

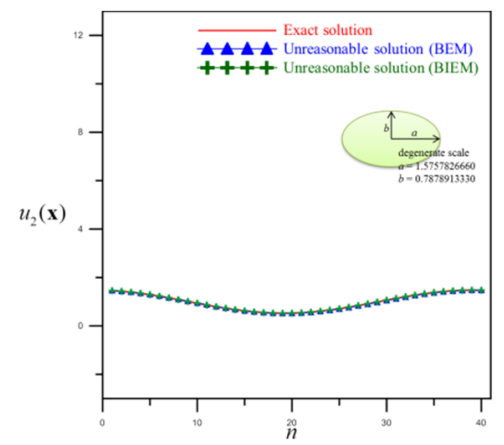

(d) The displacement $u_{2}$ (x) by using the present method

Figure 3: The results of the degenerate-scale case $(b=0.7878913330)$ for an elliptical domain.

shown in figure 4. The numerical results are unreasonable for the case of the rigid body translation. In contrast, numerical results are acceptable for the case of the rigid body rotation. Therefore, we may wonder why the numerical results approach the exact solutions in the ill-posed system when the size of domain is a degenerate scale. We employed the SVD technique to find the MPF of the mode for the near-zero minimum singular value.

When the number of boundary elements is 20 , the minimum singular value of the influence matrix is $6.3 \times 10^{-6}$ in this case. The MPFs of the mode for the singular values are shown in figure 5, where $\beta_{n}^{(1)}$ and $\beta_{n}^{(2)}$ are the $n$th MPFs corresponding to the $n$th singular value for the case of the rigid body translation and the case of the rigid body rotation, respectively. When the size of the elliptic domain is the degenerate scale $(b=0.787891333)$, the MPFs corresponding to the near-zero minimum singular value $\left(\sigma_{1}=6.3 \times 10^{-6}\right)$ are $\beta_{1}^{(1)}=-4.34859$ and 


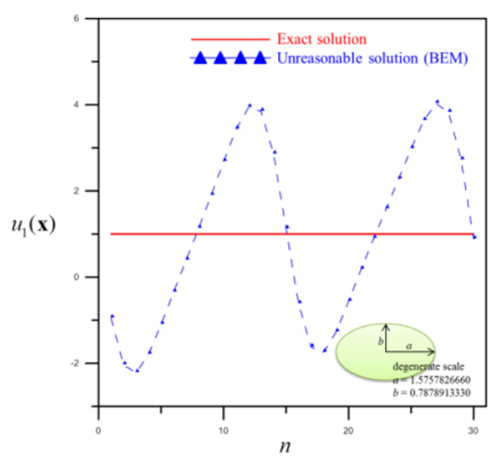

(a) The displacement along the $x_{1}$ direction (rigid body translation)

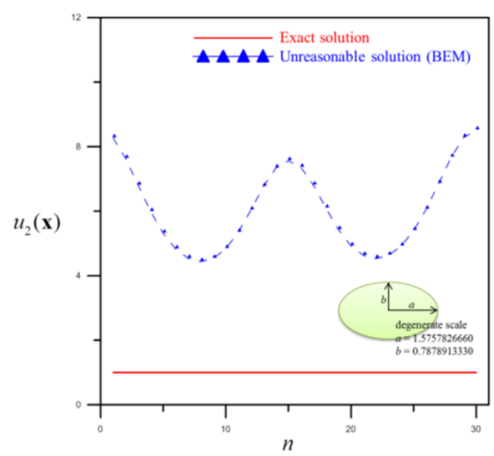

(b) The displacement along the $x_{2}$ direction (rigid body translation)

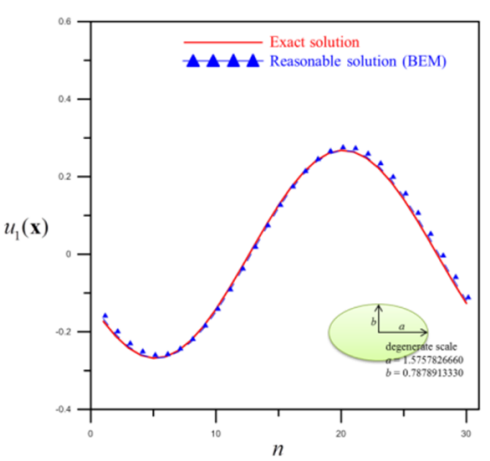

(c) The displacement along the $x_{1}$ direction (rigid body rotation)

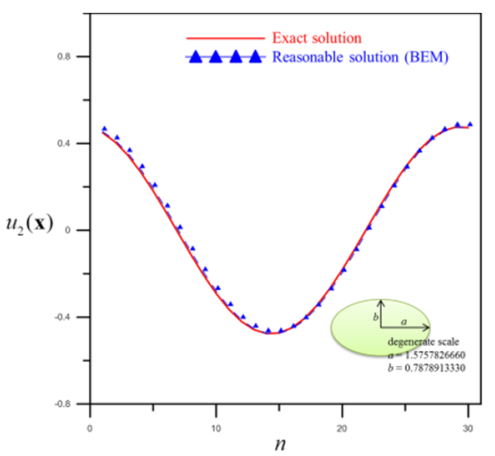

(d) The displacement along the $x_{2}$ direction (rigid body rotation)

Figure 4: The results of the degenerate-scale case $(b=0.7878913330)$ for an elliptic domain subjected to two boundary conditions by using the conventional BEM.

$\beta_{1}^{(2)}=0$. Two different MPFs are used to explain why the results of the displacement solution fail by using the conventional BEM to solve the degeneratescale problem if the solution contains the constant term. However, results of the degenerate-scale problem subject to the condition of the rotation are acceptable.

The aforementioned example including the boundary conditions of the rigid body translation or rotation is very simple for 2-D elasticity problems. However, the conventional BIEM fails to solve because the mathematical model of integral equation formulation is not complete. This simple test (rigid body translation) is used to examine the sufficient and necessary BIEM to ensure a unique solution as the patch test (constant strain) for the FEM. 


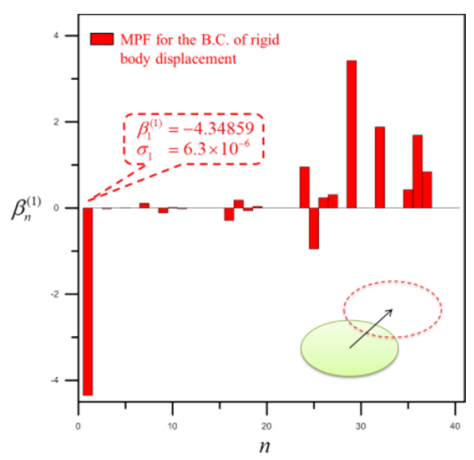

(a) Boundary condition of a rigid body translation

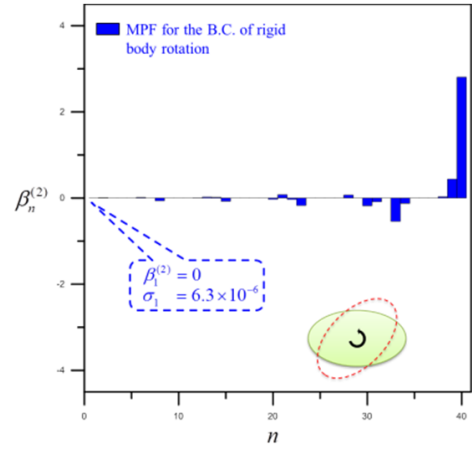

(b) Boundary condition of a rigid body rotation

Figure 5: The modal participation factors for various boundary conditions when the size of an ellipse is the degenerate scale $(b=0.7878913330)$.

\section{Conclusions}

A simple test of the BEM/BEIM for the rigid body translation and rotation problems was proposed in this paper. An example with the specified rigid body displacement boundary condition is the basic test for the BIEM/BEM as the patch test for the FEM. Two tests are compared in table 1. For the degenerate scale of the 2-D elasticity problem, we revisited the issue of the sufficient and necessary BIEs. We examined the role of the adding rotation term in Hu's sufficient and necessary BIEs for the degenerate-scale problem by using the degenerate kernel and the MPFs. Based on analytical results, the range deficiency only occurs in the constant term of the solution space instead of the rigid body rotation for the interior problem. In the numerical implementations, we investigated the MPF for various boundary conditions when the size of domain is a degenerate scale. It is found that the MPF corresponding to the near-zero minimum singular value is zero when the boundary condition is a rigid body rotation without containing any translation. Therefore, the additional rotation term is not necessary to be included in the regularized BIE for the interior degenerate-scale problem. We extended the Fichera's idea to add a constant and a corresponding constraint for the rank promotion. The necessary and sufficient BEM/BIEM to ensure a unique solution was examined. Finally, we successfully enriched the range deficiency by adding a constant term in the BIEM for the problem with a degenerate scale.

Table 1: The comparison of the simple test for the BEM and the FEM.

\begin{tabular}{|c|c|c|}
\hline & BEM/BIEM & FEM \\
\hline Test & Rigid body test & Patch test \\
\hline Boundary condition & $\begin{array}{c}\text { Rigid body translation } \\
\text { and rigid body rotation }\end{array}$ & $\begin{array}{c}\text { Uniaxial stress to check } \\
\text { the constant strain }\end{array}$ \\
\hline
\end{tabular}




\section{References}

[1] Feng, K., Differential vs. integral equations and finite vs. infinite elements. Mathematica Numerica Sinica, 2, pp. 100-105, 1980.

[2] $\mathrm{Yu}, \mathrm{D} . \mathrm{H}$., Natural boundary integral method and its applications. Science press/Kluwer, Beijing/New York, 2002.

[3] Chen, J.T., Han, H., Kuo, S.R. \& Kao, S.K., Regularization methods for illconditioned system of the integral equation of the first kind with the logarithmic kernel. Inverse Problems in Science and Engineering, 22, pp. 1176-1195, 2014.

[4] Chen, J.T., Lee, C.F., Chen, I.L. \& Lin, J.H., An alternative method for degenerate scale problems in boundary element methods for the twodimensional Laplace equation. Engineering Analysis with Boundary Elements, 26, pp. 559-569, 2002.

[5] Jaswon, M.A. \& Symm, G.T., Integral equation methods in potential theory and elastostatics. Academic Press, New York, 1977.

[6] Kuo, S.R., Chen, J.T., Lee, J.W. \& Chen, Y.W., Analytical derivation and numerical experiments of degenerate scale for regular N-gon domains in BEM. Applied Mathematics and Computation, 219, pp. 5668-5683, 2013.

[7] Chen, J.T., Kuo, S.R. \& Lin, J.H., Analytical study and numerical experiments for degenerate scale problems in the boundary element method for two-dimensional elasticity. International Journal for Numerical Methods in Engineering, 54, pp. 1669-1681, 2002.

[8] Chen, Y.Z., Wang, Z.X. \& Lin, X.Y., The degenerate scale problem for the Laplace equation and plane elasticity in a multiply connected region with an outer circular boundary. International Journal of Solids and Structures, 46, pp. 2605-2610, 2009.

[9] Chen, Y.Z., Approximate solution for degenerate scale problem of two rigid lines in series in plane elasticity. International Journal of Solids and Structures, 52, pp. 205-208, 2015.

[10] He, W.J., Ding, H.J. \& Hu, H.C., Degenerate scale and boundary element analysis of two-dimensional potential and elasticity problems. Computers \& Structures, 60, pp. 155-158, 1996.

[11] Vodička, R. \& Petrik, M., Degenerate scales for boundary value problems in anisotropic elasticity. International Journal of Solids and Structures, 52, pp. 209-219, 2015.

[12] Christiansen, S., On Green's third identity as a basis for derivation of integral equations. Journal of Applied Mathematics and Mechanics, 54, pp. 185-186, 1974.

[13] Chen, J.T., Chang, Y.L., Kao, S.K. \& Jian, J., Revisit of the indirect boundary element method: necessary and sufficient formulation. Journal of Scientific Computing, in Press, 2015.

[14] Hu, H.C., Ding, H.J. \& He, W.J., Equivalent boundary integral equations for plane elasticity. Science in China Series A, 40, pp. 76-82, 1997.

[15] Fichera, G., Boundary problems in differential equations. Madison (WI): The University of Wisconsin Press, 1959. 
[16] He, W.J., A necessary and sufficient boundary integral formulation for plane elasticity problems. Communications in Numerical Methods in Engineering, 12, pp. 413-424, 1996.

[17] Chen, J.T., Huang, W.S., Lee, Y.T., Kuo, S.R. \& Kao, S.K., Revisit of degenerate scales in the BIEM/BEM for 2D elasticity problems, Mechanics of Advanced Materials and Structures, Accepted, 2015.

[18] Chen, J.T., Huang, W.S., Lee, Y.T. \& Kao, S.K., A necessary and sufficient BEM/BIEM for two-dimensional elasticity problems, Submitted, 2015. 